\title{
Puentes entre reformas curriculares y propuestas docentes: reconstrucción del aula de Lengua y Literatura
}

\author{
MICAELA LORENZOTTI Universidad Nacional del Litoral-CONICET, Argentina / lorenzottimicaela@gmail.com
}

\section{Resumen}

A partir de la sanción de la Ley de Educación Nacional N ${ }^{\circ}$ 26.206 en el año 2006 , se inició un proceso de reestructuración curricular de la que los contenidos del aula de lengua y literatura no quedan exentos. Los cambios curriculares se implementan en todos los niveles de estructuración formal del curriculum: nacional, jurisdiccional e institucional, y en cada uno de esos niveles los contenidos de lengua y de literatura son sometidos a cambios. Pero, ¿qué decisiones toman los docentes en relación con estos cambios? La respuesta no es unísona. Concebimos al «docente autor del curriculum» como aquel consciente de las transformaciones curriculares, que las analiza críticamente y establece los puentes entre las reformas que giran en torno de sus objetos de enseñanza y sus aulas de lengua y literatura. Este trabajo busca reconstruir esos puentes que elaboran los docentes. Para ello, se indaga en las propuestas de aulas de lengua y literatura en relación con los contenidos oficiales enmarcadas en la Ley de Educación Nacional Nº 26.206.

Palabras clave: aulas de lengua / contenidos gramaticales / reelaboraciones curriculares / docente autor del curriculum / reforma curricular

\section{Path between curricular improvements and academic proposals: rebuilding Language and Literature classroom \\ Abstract}

Since the enactment of the National Education law ( ${ }^{\circ}$ 26,206 ) in 2006 , a curricular rebuilding process has been initiated within the Argentinian educational system. Language and Literature contents have not been exempt from this process. Curricular changes are implemented in all levels of formal structuring of the curriculum: national, jurisdictional and institutional, and in each one of those levels Language and Literarure contents are submitted to changes. But, what kind of decisions do teachers make regarding these changes? The answer is not unique. We regard «the teacher author of the curriculum», as the one aware of the curricular transformations, who critically analyses and establishes connections between the reforms related to the teaching objects and Language and Literature classrooms. This paper seeks to restore those connections created by teachers. In order do so, teaching proposals within Language and Literarure classes and their relation to the official contents framed on the National Education law are delved into.

Key words: Language classrooms / grammatical contents / curriculum reelaboration / teacher (as) author of the curriculum / curriculum reform

Fecha de recepción: 30/11/2017; fecha de aceptación: 26/2/2018

Para citar este artículo: Lorenzotti, Micaela (2018). «Puentes entre reformas curriculares y propuestas docentes: reconstrucción del aula de Lengua y Literatura». El taco en la brea 7 (diciembre-mayo), 63-85 Santa Fe, Argentina: UNL. DOI: https://doi.org/10.14409/tb.voi7.7355 
La historia está hecha tanto de continuidades como de rupturas; y el conocimiento de esas continuidades y rupturas da un sentido distinto a los discursos y a las prácticas actuales. Maite Alvarado

\section{Presentación}

La enseñanza de la lengua y la literatura en la escuela secundaria argentina ha sido foco de múltiples debates en los últimos años. Las instituciones educativas son los escenarios en los que pueden leerse las diversas repercusiones que las reformas educativas traen aparejadas. Dentro de esos escenarios macro, las aulas son los espacios particulares en los que se manifiestan, ya sea por la continuidad o por la ruptura, las propuestas de las reformas curriculares oficiales.

Así entonces, las aulas son el escenario de las prácticas concretas, y en ellas confluyen las propuestas curriculares oficiales, los mandatos institucionales y las decisiones docentes. En este contexto, el docente es el eslabón principal en esa cadena de reelaboraciones curriculares: pone en jaque las propuestas, las reordena, las reelabora, las reconstruye, las hace propias y elabora su propuesta de aula particular.

Entendemos a la Didáctica, a partir de los postulados de Litwin y Camilloni, como una disciplina que se ocupa de las prácticas de la enseñanza no aisladas sino significadas en sus contextos sociohistóricos. Camilloni explica que la didáctica es «una disciplina teórica que se ocupa de estudiar (...) las prácticas de la enseñanza, y que tiene como misión describirlas, explicarlas y fundamentar y enunciar normas para la mejor resolución de los problemas que estas prácticas plantean a los profesores» (22). Por su parte, Litwin entiende a la didáctica como la «teoría acerca de las prácticas de la enseñanza significadas en los contextos sociohistóricos en que se inscriben» (94).

La didáctica se preocupa por dar respuestas a cuestiones relacionadas con las prácticas de enseñanza pero sin diferenciar campos de conocimiento, niveles de educación, edades o tipos de establecimientos. Son las didácticas específicas las encargadas de desarrollar campos sistemáticos del conocimiento didáctico que se caracterizan por tomar como punto de partida una delimitación de regiones particulares del mundo de la enseñanza. En esta instancia el criterio de diferenciación de regiones que nos interesa desarrollar es el de las disciplinas, el de las didácticas específicas.

Es necesario resaltar que a la disciplina lengua y literatura le conciernen dos objetos de enseñanza. En concordancia con lo que sostiene Bombini consideramos que es necesario pensar en una «didáctica de doble objeto» teniendo en cuenta que la especificidad del objeto lengua y la especificidad del objeto literatura proponen un tratamiento didáctico particular, lo que implica que debemos devolverle a cada disciplina su identidad.

Si tenemos en cuenta entonces esta particularidad debemos aclarar que nuestro análisis se concentra exclusivamente en problemas vinculados con la didáctica de la lengua. Por esta razón, se indaga en las «aulas de lengua» (Gerbaudo 2011), categoría con la que nos referimos a:

no (...) sólo el diseño didáctico de la clase sino el conjunto de decisiones previas que se ponen en juego en cada una de esas actuaciones a lo largo de un período lectivo: la selección de contenidos, de materiales, los corpus (...), los envíos (...), el diseño de evaluaciones, la configuración didáctica (Litwin) de las clases. Este concepto llama la atención sobre el carácter artesanal y complejo de cada propuesta didáctica dado que cada grupo particular de alumnos requiere una re-selección y una composición didáctica de los contenidos pautados por los Ministerios (nacionales tanto como provinciales). (20) 
Acotando entonces la premisa que abre este apartado, la enseñanza de la lengua en la escuela secundaria argentina ha sido foco de múltiples debates en los últimos años. En el año 2006 se sancionó la Ley de Educación Nacional N²6.206 (de aquí en adelante LEN). A diferencia de lo que ocurrió con la Ley Federal de Educación N²4.195 (de aquí en adelante LFE) en la que los contenidos propuestos para cada disciplina no fueron consultados con la comunidad educativa en general, en el Nivel Jurisdiccional Santa Fe se crearon borradores de las propuestas curriculares para cada área que los docentes discutieron en reuniones institucionales. El primer documento curricular oficial de la jurisdicción Santa Fe para el Nivel Secundario enmarcado en la LEN se denomina Orientaciones Curriculares del área de Lengua y Literatura de la Jurisdicción Santa Fe, versión 2012, (de aquí en adelante OCLyL 2012) y de su lectura se desprende una vieja problemática que acecha al área Lengua: la enunciación de los contenidos gramaticales en el espacio curricular lengua y literatura.

Dentro de los contenidos gramaticales centramos nuestra atención en el contenido gramatical verbo (de aquí en adelante CGV). Consideramos que «el verbo es el nudo a partir del cual se establece la red de relaciones que conforman el mensaje» (Defagó y Guglielmelli). Defagó y Guglielmelli explican que la información semántica del verbo es un aspecto que se puede manipular conscientemente y desde la que se puede partir en el análisis. Por lo tanto desde el conocimiento semántico que tienen los alumnos podrían repensar, explicar y determinar cómo se organiza la oración en torno del verbo, es decir, qué sintagmas deben ensamblársele según qué tipo de verbo sea, qué papel semántico debe desempeñar cada uno de esos sintagmas en la oración y en qué estructuras sintácticas deben ordenarse.

Coincidimos con Carrió (2005) en que «la enseñanza de la gramática (...) es un medio y no un fin, es un paquete de herramientas y no un punto de llegada». A partir de la enseñanza del CGV en el «aula de lengua» los alumnos podrían reflexionar sobre la construcción textual, porque, como afirma Demonte en la construcción de los textos ciertas opciones gramaticales iniciales como la selección de tiempos verbales determinan la estructura del texto, «los tiempos verbales son fenómenos gramaticales y desde allí llegan al texto, y no viceversa» (183).

$\mathrm{El}$ conocimiento sobre el CGV nos permite reflexionar sobre los requerimientos de su formación y combinación, nos permite construir generalizaciones sobre procesos derivativos a partir de la morfología, «el saber sobre las correlaciones verbales permite evaluar su uso adecuado en los textos, y por lo tanto, la coherencia de éstos» (Otañi y Gaspar 2001:103), nos permite explicitar las relaciones de concordancia entre el sujeto y el verbo; la exigencia de una estructura que funcione como complemento en los verbos transitivos. Desde la perspectiva sintáctica resulta importante distinguir entre elementos obligatorios y opcionales y saber cuáles son las relaciones semánticas que se establecen entre las categorías que constituyen una oración (Llambí de Adra). Establecer la relación entre la categoría verbo y la categoría nombre implica repensar los rasgos morfológicos y sintácticos que se expresan en la concordancia sujeto-predicado:

las cuestiones relativas a las clases de palabras no son pertinentes sólo para la gramática sino que inciden directamente en el uso de la lengua y su desconocimiento está en la base de muchos errores comunes de los estudiantes, estas nociones (...) son de suma utilidad para mejorar la producción de textos en los diferentes ciclos de escolarización. (Giammatteo y Albano:69) 
A partir de esta supuesta ausencia del CGV en los documentos oficiales es que indagamos en las propuestas concretas de los docentes. La categoría que guía el análisis de las propuestas docentes gira en torno a la noción del «docente como autor del curriculum» (Gerbaudo 2006) porque entendemos que el docente es autónomo en tanto que es quien trabaja activamente en la construcción de su propuesta de enseñanza:

Los profesores no son simples receptores pasivos de los enunciados proferidos desde los espacios oficiales sino que han sido formados (o debieran haber sido formados) para intervenir como sujetos activos y críticos en el proceso de determinación curricular, aprovechando los intersticios existentes entre las propuestas de cada uno de los sectores. (151)

De este modo, siguiendo a Giroux concebimos al docente en tanto «intelectual transformativo» y no como un mero técnico que se encarga de llevar adelante dictámenes decididos por expertos ajenos a la realidad del aula. Para pensar esta selección que los docentes hacen a la hora de configurar sus "propuestas de aulas de lengua» se hace indispensable recuperar la noción de curriculum. Siguiendo a De Alba entendemos por curriculum a «la síntesis de elementos culturales (conocimientos, valores, creencias, costumbres) que conforman una propuesta político-educativa pensada e impulsada por diversos grupos y sectores sociales cuyos intereses son diversos y contradictorios» (38-39). Esos elementos culturales que son seleccionados para conformar una determinada propuesta político-educativa se incorporan al curriculum a través de sus aspectos formales-estructurales pero además por medio de las «relaciones sociales cotidianas en las cuales el curriculum formal se despliega, deviene práctica concreta» (39).

De Alba explicita que los grupos dominantes de una sociedad son quienes consideran a ciertos elementos culturales como valiosos para ser incorporados en el curriculum. Esto implica que otros elementos culturales, no considerados valiosos por esos grupos dominantes, queden excluidos. Este proceso de selectividad y de exclusión que se efectúa en diferentes niveles de la elaboración del curriculum es lo que Morzán denomina como «curriculum nulo». El «curriculum nulo» está conformado por «aquellos conocimientos que no penetran en el discurso escolar o que en todo caso si lo hacen, se introducen de un modo banal» (107).

Esta propuesta se inició con la supuesta ausencia de contenidos gramaticales en los documentos curriculares oficiales de la jurisdicción Santa Fe. Siguiendo a Flinders, Noddings y Thornton creemos que prestar atención al «curriculum nulo» nos impulsa a reexaminar las metas y los criterios de selección a la luz del contenido. Es mediante el proceso que De Alba denomina "proceso de determinación curricular» que se dan las luchas, negociaciones o imposiciones de diferentes grupos y sectores que determinan un curriculum en sus aspectos centrales. En este proceso los sujetos del curriculum cumplen diferentes funciones:

- Los sujetos de la determinación curricular: son los que están interesados en determinar los rasgos básicos o esenciales de un curriculum particular (el Estado por ejemplo).

- Los sujetos del proceso de estructuración formal del curriculum: son los que en el ámbito institucional escolar le otorgan forma y estructura al curriculum de acuerdo a los rasgos centrales perfilados en el proceso de determinación curricular. (Aquí es donde se elabora el plan de estudios). 
- Los sujetos del desarrollo curricular: son los que convierten en práctica cotidiana un curriculum. (Los profesores y alumnos).

Nuestro interés está centrado en los sujetos de la determinación curricular y los sujetos del proceso de estructuración formal del curriculum porque interesa poner en relación el modo en el que las instituciones educativas reelaboran los contenidos propuestos por los documentos oficiales.

Remitiéndonos a Puiggrós entendemos que las prácticas docentes no se encuentran aisladas sino que dialogan directamente con el contexto del que forman parte y con la historia general de la educación, con los diferentes procesos que fueron, y aún siguen, configurando lo que hoy es el sistema educativo argentino. Es decir, las prácticas de enseñanza varían de acuerdo a ese contexto social del cual no sólo surgen sino que cobran sentido, contexto que cada vez se presenta como único y variable. De allí que los factores que intervienen e inciden en las prácticas sean diversos porque se relacionan justamente con ese contexto que las envuelve y las determina como tales.

A la hora de indagar en las ofertas docentes entendemos que las mismas están guiadas por el concepto de «buena enseñanza» que propone Fenstermacher:

El adjetivo «buena» no es sinónimo de «con éxito», (...). Por el contrario, en este contexto la palabra buena tiene tanto fuerza moral como epistemológica. Preguntar qué es buena enseñanza en el sentido moral equivale a preguntar qué acciones docentes pueden justificarse basándose en principios morales y son capaces de provocar acciones de principio por parte de los estudiantes. Preguntar qué es buena enseñanza en el sentido epistemológico es preguntar si lo que se enseña es racionalmente justificable y, en última instancia, digno de que el estudiante lo conozca, lo crea o lo entienda. (158)

Otañi y Gaspar (2001) anunciaban que en la escuela la gramática «incomodaba». Las autoras pensaron el lugar de la gramática en la escuela desde cuatro versiones que, a modo de metáfora del enamoramiento, sintetizan el modo en que los docentes se relacionan con la gramática: i) la atribución de un papel protagónico; ii) la elaboración de su acta de defunción; iii) la asignación de un papel «extra» y iv) la ubicación como protagonista junto con otros saberes de la lengua y la literatura.

El recorrido propuesto en este trabajo busca respuestas a los siguientes interrogantes iniciales:

(i) ¿qué lugar ${ }^{2}$ ocupa la categoría gramatical verbo en los documentos curriculares oficiales de la LEN?;

(ii) ¿qué lugar le asignan los docentes como «autores del curriculum» (Gerbaudo 2006) al contenido gramatical verbo en sus propuestas de aulas de lengua?; 3

(iii) ¿cómo se articulan las propuestas de aulas de lengua con las propuestas educativas oficiales?

\section{Un poco de historia: los contenidos en la LFE}

Desde la implementación de la LFE los contenidos curriculares se desarrollan en tres niveles de elaboración:

(i) Nivel Nacional: los contenidos son propuestos por el Ministerio de Educación de la Nación. 
(ii) Nivel Jurisdiccional: los contenidos propuestos por el Nivel Nacional son reelaborados en cada Ministerio provincial.

(iii) Nivel Institucional: cada institución educativa adecua los contenidos reelaborados por el Nivel Jurisdiccional a sus contextos particulares.

Si bien nuestro interés está centrado en la nueva reforma curricular impulsada por la LEN, para iniciar este recorrido y poder responder a los tres interrogantes iniciales se hace imprescindible responder primeramente al siguiente interrogante: ¿cuál es el lugar de los contenidos gramaticales en las reelaboraciones curriculares de la LFE?

De la lectura de los documentos oficiales de la LFE se observa que en este cambio educativo se le declaró el «acta de defunción» (Otañi y Gaspar 2008) a la reflexión gramatical más allá del texto dentro del área lengua.

El nuevo objetivo del área era que «el alumno aprenda a adecuar y controlar los recursos lingüísticos en prácticas discursivas de complejidad creciente» (Diseño Curricular Jurisdiccional Tercer Ciclo EGB Lengua 1999:97) y en consonancia con dicho objetivo la secuenciación curricular subordina a los contenidos gramaticales al lugar de su reconocimiento en la construcción de los formatos de cada tipo de texto.

En el documento Orientaciones Didácticas para el Tercer Ciclo EGB de Lengua se le dedica un apartado al interrogante ¿Es necesario enseñar gramática? Nuestra inquietud reside en por qué es necesario, en la primera propuesta curricular oficial desde la Ley de Educación Común N 1420, formularse esa pregunta. Más aún, por qué es necesario, en un documento oficial, justificar las respuestas a esa pregunta. Lo que se defiende en ese apartado es la idea de que por muchos años la gramática se enseñó de manera aislada, descontextualizada y «divorciada de las prácticas de comprensión y producción oral y escrita» (54) y que por ello hay que replantearse su inclusión curricular.

La sugerencia que instalan los Documentos Jurisdiccionales de Santa Fe de la LFE (de aquí en adelante DJ) es la enseñanza de la gramática en función de mejorar el desempeño en la lectura y escritura de los textos. La tendencia explícita de los DJ es la de «jerarquizar el contenido comunicativo y textual en la enseñanza de la Lengua, sin descartar del todo los contenidos gramaticales, aunque aparezcan escasamente integrados con los anteriores» (Ciminari y Kocak:170).

En otro texto oficial, «Documento de apoyo para $8^{\circ}$ año, Lengua», se pone de manifiesto la inestabilidad de los argumentos que los «sujetos de determinación curricular» (De Alba) adoptan con relación a lo que se debe enseñar en las clases de lengua. Desde los mismos documentos oficiales de la LFE se pone en duda, en jaque, en discusión — discusión que simula ser colectiva— la enseñanza de la gramática en la disciplina lengua (y literatura). A esto se suma la inminente aparición en los Contenidos Básicos Comunes de Nivel Nacional (de aquí en adelante $\mathrm{CBC}$ ) de contenidos que la mayoría de los docentes conocía parcialmente o desconocía. Tal como afirma Cortés, en la transición de la LFE los docentes de lengua tuvieron que interiorizarse en el nuevo marco teórico que tenía como objeto de estudio al texto o discurso y esta interiorización fue un gran desafío porque «la urgencia por implementar los nuevos contenidos curriculares dificultó llevar a cabo esa transposición [didáctica], ya que no se habían concretado las mediaciones necesarias para acompañar a los docentes en el procesamiento de esos cambios, y sus consecuentes aplicaciones en el aula» (115).

Advertimos que en los CBC los contenidos gramaticales propuestos se presentan aislados de 
los tipos de textos. Son, aunque escasos, un contenido de reflexión en sí mismos. No obstante esto, esos contenidos gramaticales no son recuperados en los contenidos de los DJ. A nivel jurisdiccional si bien se propone como objetivo del área lengua propiciar la reflexión metalingüística del alumno, se espera lograrlo a partir de la sistematización de los tipos textuales, dejando de lado a la lengua como objeto de estudio y reflexión más allá de la unidad textual.

\section{La desorientación: la imprecisión curricular de la LEN}

Luego del breve recorrido por las reformulaciones de la LFE volvamos ahora al primero de nuestros interrogantes: ¿Qué lugar ocupa la categoría gramatical verbo en los documentos curriculares oficiales de la LEN?

Para realizar este análisis nos centramos en los documentos curriculares de nivel nacional denominados Núcleos de Aprendizajes Prioritarios (de aquí en adelante NAP) y en los documentos curriculares de nivel jurisdiccional denominados Orientaciones Curriculares (de aquí en adelante OC).

Los NAP se definen como un conjunto de saberes centrales, relevantes y significativos para ser incorporados como objetos de enseñanza. Se los considera como núcleos organizadores de la enseñanza y están secuenciados anualmente. El cuadernillo correspondiente al Ciclo Básico de la Educación Secundaria contiene la propuesta de contenidos para primer y segundo año. El documento perteneciente al área lengua está secuenciado en cuatro ejes:

(i) En relación con la comprensión y la producción oral.

(ii) En relación con la lectura y la producción escrita.

(iii) En relación con la literatura.

(iv) En relación con la reflexión sobre la lengua (sistema, norma y uso) y los textos.

$\mathrm{Al}$ analizar los NAP para primer año del Ciclo Básico de la Educación Secundaria (2011) encontramos que en la primera parte del eje iv «En relación con la reflexión sobre la lengua.... los contenidos gramaticales están vinculados con el análisis de textos que se enumeraron como núcleos en los ejes anteriores:

-La reflexión sistemática, con ayuda del docente, sobre distintas unidades y relaciones gramaticales y textuales distintivas de los textos trabajados en el año, así como en situaciones específicas que permitan resolver problemas, explorar, formular hipótesis y discutirlas, analizar, generalizar, formular ejemplos y contraejemplos, comparar, clasificar, aplicar pruebas, usando un metalenguaje compartido en relación con:

(...)

* La narración (su estructura prototípica, distintas funciones de la descripción y el diálogo en el relato; personas gramaticales y tipos de narrador; los tiempos verbales propios del relato y sus correlaciones: pretérito perfecto simple y pretérito imperfecto para dar cuenta de los hechos, pretérito pluscuamperfecto para narrar hechos anteriores al tiempo del relato, presente y pretérito imperfecto para presentar el marco o describir personajes u objetos presentes en el diálogo, condicional para el futuro de los hechos del relato; conectores temporales, causales y consecutivos);

* Los textos de divulgación (el presente para marcar la atemporalidad; los adjetivos descriptivos y las nominalizaciones. Organizadores textuales y conectores; procedimientos: ejemplos, definiciones, comparaciones, paráfrasis, descripciones y narraciones, y recursos gráficos); 
(...)

* Las distintas formas de introducir la palabra del otro: estilo directo e indirecto y verbos introductorios (ampliación del repertorio de verbos de decir), correlaciones en el estilo indirecto). (24-25)

No obstante en un primer momento los contenidos gramaticales están estrechamente vinculados con el contenido textual, la enumeración de contenidos avanza y aparecen listados contenidos de gramática oracional desligados de contenidos de gramática textual:

\footnotetext{
* los constituyentes de las oraciones a través de pruebas (cambio de orden, sustitución, interrogación);

* clases de palabras: sustantivos, adjetivos, verbos, preposiciones, adverbios, artículos y pronombres (personales, posesivos, demostrativos e interrogativos y exclamativos);

* palabras variables e invariables; categorías morfológicas nominales (género y número) y verbales (tiempo, modo y persona). Concordancia;

* la construcción sustantiva y verbal (núcleo y modificadores) y funciones sintácticas en la oración simple;

* relaciones de significado entre las palabras: sinónimos, antónimos, hiperónimos, hipónimos, para la ampliación del vocabulario y para inferir el significado de las palabras desconocidas; como procedimiento de cohesión, como recurso de estilo;

* formación de palabras (morfología derivativa: sufijación, prefijación, composición) y algunos casos de etimología para la ampliación del vocabulario, para inferir el significado o la ortografía de alguna palabra. $(25-26)$
}

De la enumeración de los contenidos del eje iv se desprende que en la elaboración curricular del nivel nacional la reflexión gramatical está diagramada a partir de la incorporación de contenidos relacionados con la gramática textual como así también de la gramática oracional. Se proponen contenidos relacionados con la morfología, la sintaxis y la semántica.

Perkins se pregunta ¿qué esperamos de la educación? Y la respuesta a la que arriba es «todo»; pero si pudiéramos decidir qué del todo preferimos de ella, la respuesta se formularía de otra manera. Preferimos que permita un «conocimiento generador; es decir, conocimiento que no se acumula sino que actúa» (18).

Creemos que en el eje iv de los NAP se listan contenidos que pueden convertirse en conocimiento generador, generador de reflexiones lingüísticas e indagaciones no sólo sobre los usos del conocimiento gramatical en función de la construcción de textos sino también sobre la estructura del objeto de estudio del área, esto es, la estructura de la lengua.

En la secuencia didáctica de la LFE la reflexión gramatical que se proponía estaba al servicio de la escritura y, como lo explican Otañi y Gaspar «incluso se plantea su posterioridad temporal: en cada clase, primero se escribe y luego se reflexiona sobre alguna cuestión problemática del texto producido» (2001:94). En los contenidos de la LEN se advierte que la reflexión gramatical no está al servicio de la escritura ni temporalmente posterior a ella sino que forma parte tanto de la reflexión sobre la estructura de los textos como de la reflexión sobre la estructura de la lengua. Este es un aporte importante en el cambio curricular y una continuidad con relación a los CBC de la LFE. En el nivel nacional de la LFE se contemplaban contenidos vinculados con la gramática oracional. Parecería que en el primer nivel de elaboración curricular no se discute sobre el por qué y para qué enseñar gramática en la escuela, lo que se evidencia en los contenidos. 
El segundo nivel de elaboración curricular es el jurisdiccional. Este nivel elabora su propuesta curricular a partir de los NAP del nivel nacional. Para llevar adelante el análisis se trabajó sobre el primer documento curricular jurisdiccional de la provincia de Santa Fe, la versión 2012 correspondiente a las Orientaciones Curriculares del Ciclo Básico de la Educación Secundaria para el área Lengua y Literatura (OCLyL 2012). ${ }^{4}$

La versión 2012 es una versión piloto que fue efectivamente implementada en las escuelas durante el período académico 2012. De las evaluaciones sobre esta prueba piloto se redefinió la segunda versión del año 2013 implementada desde el inicio de ese mismo año.

En el ciclo lectivo 2012 el Ministerio de Educación de la Provincia de Santa Fe acercó a las instituciones educativas la primera versión de las OCLyL 2012. Esta versión circuló durante un año en las instituciones educativas, período durante el cual fue revisada a partir de la conformación de una Comisión $a d h o c^{5}$ y de los aportes y sugerencias que realizaron los docentes en las reuniones institucionales llevadas a cabo en el transcurso del año 2012. En febrero del año 2013 el ministerio acercó a las instituciones la nueva versión de las Orientaciones Curriculares para el Ciclo Básico de la Educación Secundaria (OCLyL 2013).

Interesa describir aquí las OCLyL 2012 porque es sobre la base de ese documento que los docentes seleccionados como muestra elaboraron sus primeras propuestas de aulas de lengua enmarcadas en el cambio curricular de la LEN.

En las OCLyL 2012 se fundamenta que el eje de la asignatura son las cuatro habilidades, ya instaladas desde la LFE, es decir: hablar, escuchar, leer y escribir; y el sujeto del aprendizaje es concebido como un «sujeto de acción verbal»: "Aprender Lengua y Literatura implica hablar, escuchar, leer y escribir la lengua que nos pertenece y nos constituye en sujetos de acción verbal, desde la que pensamos el mundo y, a la vez, nos pensamos en nuestra relación con él y con los otros sujetos en un espacio histórico» (31).

La concepción de la propuesta jurisdiccional del sujeto de aprendizaje en términos de «sujeto de acción verbal» genera algunas confusiones. Se le atribuye a la lengua distintas funciones, pero, si bien la lengua es una herramienta de representación, con la que nombramos el mundo que nos rodea, difícil es asegurar que el sujeto piensa su relación con el mundo y se piensa en su relación con los demás sujetos a través de la lengua. Difícil es saber de qué manera piensa el ser humano, diferente es afirmar de qué manera el ser humano exterioriza su pensamiento.

El eje de los contenidos del área es la actividad verbal del alumno, un «sujeto de acción verbal», un sujeto que habla, escucha, lee y escribe. En la fundamentación del espacio se aclara, tal como ocurrió en los DCJ de la LFE, que se deja de lado la concepción de la lengua como un objeto teórico de reflexión, como un sistema por describir a través de sus unidades. Sin embargo, el modelo didáctico propuesto contempla la descripción de unidades que conforman a los textos:

El modelo didáctico parte de las acciones verbales de los alumnos al comprender y producir textos orales y escritos. Desde la inferencia hasta la sistematización de las regularidades observables en los textos, empezando con los géneros textuales y los tipos de discurso en situaciones comunicativas, para continuar con los mecanismos de textualización y luego la organización de las frases y las unidades morfosintácticas. (38)

Como se evidencia en la cita, la reflexión sobre las unidades gramaticales que constituyen un texto se realiza en un segundo momento del trabajo con los géneros textuales. Con esta secuencia 
didáctica se argumenta que la reflexión gramatical no sea el eje de la unidad sino uno más de los múltiples contenidos que los alumnos aprenden: «tanto en el texto oral como escrito, las variaciones gramaticales son la expresión de elementos significativos para la comprensión y la producción, ya no "niveles" de análisis sin relación con estos procesos» (39).

En el documento trabajado el objeto disciplinar lengua está pensado desde una mirada cultural. Se entiende que la lengua le permite al sujeto ser social, interactuar con el medio, comunicarse y pensar. No obstante esto, en un breve pasaje inicial se reconoce que «los hablantes ingresan al ámbito escolar con conocimientos lingüísticos implícitos y será la escuela la que se encargue de desarrollar capacidades metalingüísticas que les permitan el "desarrollo" del pensamiento» (31).

El problema que subyace en la conceptualización anterior es que desarrollar las capacidades metalingüísticas del hablante permite el desarrollo de su conocimiento sobre su lengua, no de su pensamiento. De esta manera, desde los documentos se concibe al objeto lengua como objeto de vehiculización cultural y como objeto de estudio en sí mismo. Si bien en la fundamentación del documento se postula esta doble concepción del objeto disciplinar, luego esa doble caracterización es reducida a partir de fundamentos que señalan que la lengua en el aula es concebida de manera instrumental, al igual que en los DC de la LFE.

De estos fundamentos se desprenden las siguientes premisas:

(i) Se concibe, de manera textual en los documentos, al espacio curricular como trasversal e instrumental. Esto deja entrever que no se piensa al objeto lengua como objeto de estudio sino que se lo piensa como constructor de competencias que le sirven al alumno «para...». En este caso, para construir las competencias que son necesarias en todos los espacios curriculares: «de allí que el estudio y reflexión lingüísticos sean imprescindibles para favorecer el desarrollo de las capacidades humanas que permiten interactuar en el mundo con un pensamiento rico y flexible» (32). El objetivo de estudiar y reflexionar lingüísticamente que se declara en la fundamentación queda anulado y se concibe nuevamente al espacio, tal como se lo concebía en la LFE, con un fin utilitario. La concepción de «lengua» como objeto de estudio queda anulada y esta concepción del espacio curricular como instrumental se contrapone con el sujeto de acción verbal que se concibe desde los documentos.

(ii) Si bien se propone como fin/objetivo del área lengua y literatura la formación de hablantes competentes, el desarrollo de hábitos lectores y escritores y la afición por la lectura y la escritura, en la propuesta se explicita el distanciamiento del estudio y reflexión lingüística que involucre a los contenidos gramaticales con la siguiente justificación:

Pero los seres humanos no se comunican mediante palabras aisladas tampoco mediante oraciones aisladas, sino que tratan de transmitir significados completos, coherentes, porque necesitan ser comprendidos por otros. Los hablantes desarrollan sus actividades deslizándose entre dos vertientes fundamentales: el poder expresarse, dar forma a su pensamiento y el accionar con el otro: comprender e interpretar a su emisor. (32)

En este punto le dedicamos un breve espacio a la reflexión sobre esta cita. En la primera oración se afirma que los seres humanos no se comunican mediante palabras aisladas ¿qué se entiende aquí por palabras? Un «sujeto del habla», como se concibe en la propuesta que se analiza, no se 
comunica mediante palabras aisladas porque en la comunicación las palabras son enunciados. Lo que subyace en esta concepción es una incoherencia epistemológica e inconmensurabilidad.

Por otro lado se asume que el ser humano tampoco se comunica mediante oraciones aisladas. Aquí subyace un error conceptual: por definición la oración es una estructura con independencia sintáctica, con autonomía gramatical. El ser humano se comunica mediante enunciados, actos de habla, y no mediante oraciones. Éstas son unidades de análisis gramatical, no unidades de comunicación.

Finalmente, se concibe que el ser humano trata de transmitir significados completos, coherentes, pero ¿qué sucede con los enunciados que implican información elidida?

Se confunde lo que implica la «reflexión metalingüística» con la "comunicación humana», dos objetivos que podrían convivir en la currícula. Se concibe en el documento que el alumno no piensa en oraciones aisladas, lo que, ya explicamos, es un error conceptual, como tampoco piensa, a modo de ejemplo, en números primos, aunque sea uno de los contenidos pilares del álgebra y el razonamiento matemático y por lo tanto contenido ineludible del currículo matemático.

Coincidimos aquí con Carrió (2013), quien en su texto plantea que en la escolarización se dificulta la diferenciación entre dos dimensiones igualmente relevantes del lenguaje: la dimensión instrumental, la enseñanza de la lengua para el uso y la dimensión cognoscitiva, la enseñanza de la lengua como objeto de conocimiento. Esta dificultad entre las dos dimensiones del lenguaje es la dificultad que se evidencia en los documentos jurisdiccionales que analizamos y de allí que se reconozca en la fundamentación del área el esfuerzo por justificar el por qué no enseñar contenidos gramaticales: se está pensando, desde los contenidos, sólo en la dimensión instrumental del lenguaje.

Si el objetivo es propiciar la reflexión sobre la propia lengua (la dimensión cognoscitiva) es de ellas de donde podría partir el razonamiento. Si no se conoce, no se desmenuza, no se reflexiona sobre las unidades mínimas de la lengua, es difícil imaginar el recorrido que llevaría al estudiante a reflexionar sobre la construcción de textos complejos que le permitan «expresarse, dar forma al pensamiento» en diferentes "géneros» en tanto «sujeto de acción verbal».

En sus estudios sobre la LFE, Otañi y Gaspar (2001) argumentan que la reflexión sobre la construcción de los textos, la gramática del texto, requiere contar con conocimientos previos sobre la gramática de la lengua. Este es un camino posible para poder reflexionar sobre determinados fenómenos textuales que necesitan de un metalenguaje gramatical compartido para su explicación. Conocer ese metalenguaje y conocer los mecanismos del objeto en cuestión, el lenguaje, no implica que el alumno, «sujeto de acción verbal», se comunique mediante oraciones aisladas. La comunicación es parte de la otra dimensión del lenguaje, la dimensión instrumental.

Resulta extraño y preocupante que estas discusiones se sigan explicitando en el nuevo cambio curricular a pesar de los numerosos trabajos que desde hace años problematizan la situación de los contenidos gramaticales en las currículas durante la LFE; o, citando a las ya mencionadas autoras, «parece peligroso abandonar el proyecto de una escuela en la que el desarrollo de la curiosidad o inquietud por el conocimiento mismo también sea un objetivo relevante» (102).

En cuanto a la secuencia didáctica señalada por las OCLyL 2012, se propone que un primer nivel de abordaje de la lengua materna debe ser la actividad verbal humana-colectiva que se concreta en múltiples "géneros textuales», adecuados a diversas situaciones comunicativas. Luego, en un nivel de abordaje inferior, es cuando se propone tener en cuenta los procesos de 
estructuración sintáctica y decisiones léxicas que determina cada género: «el docente se sumerge con sus alumnos en la dimensión comunicativa del lenguaje, para guiarlos en la reflexión sobre los distintos géneros, entendidos como formas con características y estructuras definidas, creadas históricamente por comunidades» (32).

Los "géneros» son las categorías a enseñar del modelo didáctico propuesto y la justificación radica en que los géneros contemplan los tres planos: el de la acción verbal, el discursivo y el de las propiedades lingüístico-textuales. En la fundamentación se argumenta que la comprensión del funcionamiento de los géneros en su contexto le posibilita al alumno dominar la actividad verbal. Sería esperable entonces encontrar en la secuenciación de contenidos temas que desarrollen los tres planos mencionados, aunque, a primera vista, la secuencia didáctica propuesta no contempla lo discursivo, lo relacionado con la información contextual.

La secuencia didáctica propuesta queda esquematizada de la siguiente manera:

Momento 1: actividad de comprensión o producción textual de un género seleccionado.

Momento 2: selección de aspectos a enseñar en relación con las capacidades del grupo.

Momento 3: actividad de comprensión o producción textual (producción de un texto que cumpla con los requisitos constitutivos del género).

Según esta secuencia didáctica, el docente tiene demarcado un camino de enseñanza, un único camino. De los géneros que decida trabajar en el aula (tema) seleccionará los aspectos estructurales que considere relevantes enseñar sobre esos géneros (contenidos) para cerrar la secuencia con una actividad en la que esos aspectos-contenidos se pongan en juego (actividad).

Esta secuencia didáctica presenta inconvenientes para el docente que tiene a los documentos jurisdiccionales como guía para realizar su selección curricular. No se especifica qué se concibe por "géneros textuales", tampoco en qué criterios tendría que basarse el docente para hacer la selección de contenidos del «nivel de abordaje inferior» y, finalmente, ¿qué acciones implica que el docente «se sumerja en la dimensión comunicativa del lenguaje»?

La reflexión gramatical queda restringida a un segundo momento, el de la sistematización sobre las características propias de los "géneros textuales». Esta reflexión gramatical, a su vez, queda reducida a lo que el docente considere necesario trabajar con el fin de que los alumnos, en el mejor de los casos, comprendan cómo funcionan los «géneros textuales» o mejoren los problemas de escritura. En el peor de los casos se convertiría, como ocurrió en la LFE, en una mera diferenciación de rasgos «etiquetables».

Si bien en los documentos se enuncia que el objeto de estudio son los «géneros» no se los define con claridad en el documento. Esto atenta contra la propuesta porque tanto la definición superficial que se brinda como lo que aparece listado luego como contenidos se acerca más a la noción de «tipos textuales» 0 «clases textuales» que a la de «géneros»: «El carácter ilimitado de los géneros (la copla, el romance, el cuento, la novela, el chiste, el refrán, la conversación, el chat, el blog, los foros virtuales, la exposición oral, la carta familiar, la receta de cocina, la noticia policial, la nota de enciclopedia...) hace que no podamos enseñarlos todos» (37-38).

Si bien en una única línea de las OCLyL 2012 se especifica que la noción de géneros está tomada del interaccionismo sociodiscursivo no se desarrolla la concepción que subyace a dicha categoría ni tampoco se lista posible bibliografía que el docente podría consultar sobre la corriente teórica 
en que se enmarca. Nuevamente, al igual que en la LFE, el docente se encuentra con un salto teórico que debe afrontar solo.

En este sentido, Riestra explica que la noción de «tipología» ingresó a las aulas de la mano de la lingüística textual pero que sólo fue una reducción descriptiva y abstracta de los textos, un reconocimiento de formatos de textualización «situando en un segundo término la noción de contexto de producción que habían introducido las teorías pragmáticas» (6).

Bajtín define a los "géneros discursivos" como unidades que se emplean en el intercambio comunicativo:

El uso de la lengua se lleva a cabo en forma de enunciados (orales y escritos) concretos y singulares que pertenecen a los participantes de una u otra esfera de la praxis humana. Estos enunciados reflejan las condiciones específicas y el objetivo de cada una de las esferas no sólo por su contenido (temático) y por su estilo verbal, o sea por la selección de los recursos léxicos, fraseológicos y gramaticales de la lengua, sino, ante todo, por su composición o estructuración. (...) Cada enunciado separado es, por supuesto, individual, pero cada esfera del uso de la lengua elabora sus tipos relativamente estables de enunciados, a los que denominamos géneros discursivos. (248)

Pero, ¿qué se entiende por "género textual» desde el interaccionismo sociodiscursivo? Riestra explica que desde la perspectiva interaccionista social y discursiva todo texto está situado en una práctica comunicativa y social y tiene por ello una composición genérica en función de la actividad que lo produce/genera. «Es decir, "el género es un instrumento comunicativo determinado por y producido en esa práctica". Es el que permite ligar el contexto y la situación, por lo tanto es un principio organizador del texto» (9). Desde este marco, la definición de género debe remitir indisociablemente a la práctica que lo sustenta.

Ciapuscio advierte que la clasificación textual es compleja y una de las razones para que lo sea es la confusión terminológica entre «tipos textuales», «clases de textos» $\mathrm{y}$ «géneros discursivos»:

clase textual se aplica hoy a clasificaciones empíricas, tal cual son realizadas por los miembros de una comunidad lingüística, es decir, clasificaciones cotidianas que pueden mencionarse por medio de determinados lexemas condensadores del saber sobre determinada clase textual: por ejemplo, «esto es un cuento», «esto es un chiste», «esta es una descripción», «esto es un diálogo», etc. Por el contrario, «tipo textual» se concibe como una categoría ligada a una teoría para la clasificación científica de textos. Por lo tanto, los hablantes de una comunidad tienen un saber sobre clases textuales o un saber sobre estructuras textuales pero no un saber sobre tipos textuales. (25)

Los "géneros discursivos» se definen desde una perspectiva sociocultural, y los tipos y clases de textos desde una perspectiva lingüística. Si el objeto de estudio de la propuesta curricular es el «género discursivo» sería esperable que en la construcción curricular se listen como contenidos no sólo lo referido a la construcción textual, a la descripción taxonómica, sino también a las condiciones sociales de producción.

Finalmente advertimos que en las OCLyL 2012 no se explicita en el discurso un nivel de precisión deseable de un documento orientativo, es un discurso amplio en el que no se establecen los objetivos del área. Así, por ejemplo, la definición del aprendizaje del área es imprecisa: «aprender 
Lengua y Literatura no será para el alumno constituirse en lingüista o crítico literario, sino en un sujeto que piense y sienta de una manera propia» (35). ¿Cuál es esa manera propia en lengua y literatura? O ¿qué implica en educación instruir para pensar y sentir de una manera propia?

\section{El contenido ausente del Nivel Jurisdiccional}

En su estructuración, las OCLyL (2012) recuperan los cuatro ejes de los NAP. Desde una primera lectura se evidencia que los contenidos propuestos para el Primer año del Ciclo Básico de la Educación Secundaria Orientada son escasos en relación con los NAP listados en el primer nivel de elaboración curricular. A continuación se citan los contenidos de lengua para primer año propuestos en las OCLyL 2012:

\section{Eje I: «En relación con la comprensión y producción oral»}

\section{La conversación}

-Reglas generales de la comunicación.

-Estructura verbal de la conversación; propósito comunicacional, contenidos, recursos.

-Competencias comunicativas: adecuación del mensaje en virtud de la audiencia y del propósito comunicativo. (Cuándo hablar, cuándo no, de qué hablar, con quién, dónde, en qué forma).

-Estrategias discursivas para captar la atención del oyente.

-El lenguaje del cuerpo: recursos verbales y no verbales.

\section{La discusión}

-La polémica. El discurso argumentativo.

-Distinción entre «tema» $\mathrm{y}$ «problema».

-La construcción de un texto con fundamento. El punto de vista.

-Tipos o estrategias de argumentación: ejemplificación, citas de autoridad, preguntas retóricas, generalización, analogías, etcétera.

-Participantes y roles.

\section{Eje 2: Lectura y comprensión escrita}

\section{El texto expositivo}

-Estructura textual, secuencias, trama expositivo-explicativa.

-Elementos paratextuales. Estrategias de la explicación (definición, paráfrasis, etc.).

-Estrategias para lograr un texto cohesivo. Signos de puntuación, el uso de conectores.

-Objetividad en el discurso: Discurso histórico y de divulgación científica.

\section{Diarios y revistas}

-Suplementos, secciones. Editorial y carta de lectores.

-Diferencias entre el soporte papel y el formato digital.

-Características de la editorial y de la carta de lectores.

-Texto de opinión. Subjetividad.

-Lectores o destinatarios del texto.

\section{Chat, correo electrónico y mensaje de texto}

-El lenguaje mediado por las nuevas tecnologías de comunicación (TIC)

-Expresiones idiomáticas propias del género. Relaciones textuales, gramaticales, lexicales.

-El chat y sus usuarios. Variedades geográficas y sociales. Los dialectos. 


\section{Eje 4: En relación con la reflexión sobre la lengua (sistema, norma y uso) y los textos.}

SIN CONTENIDOS. (41)

Esta versión de las Orientaciones Curriculares Jurisdiccionales es importante para el docente en tanto «autor del curriculum» porque, aunque se presente con carácter de borrador, es el primer acercamiento que tiene al cambio curricular jurisdiccional desde la aprobación de la Ley en el año 2006. Pero las OCLyL son imprecisas.

El eje 4 constituye el centro de nuestro interés porque es allí donde esperaríamos encontrar listados contenidos relacionados con el CGV. No obstante esto, en el documento que analizamos este eje se encuentra vacío de contenidos; en él se lee la siguiente inscripción: «La reflexión gramatical se encuentra presente en todos los contenidos de los ejes anteriores» (42).

De aquí se desprenden dos cuestiones:

(i) Ese segundo paso de la secuencia didáctica, el de seleccionar qué contenido sobre cada género es deseable enseñar a los alumnos (en donde los docentes deberían «sumergirse») está librado a las decisiones institucionales/docentes porque en los documentos que sirven como guía/orientación no están explicitados.

(ii) Resulta difícil pensar la situación de un docente que tiene que enseñar ciertos contenidos relacionados con los textos (asumamos que estos contenidos son gramaticales) cuando los alumnos no comparten, porque no se les fue enseñado, ni el metalenguaje ni los contenidos mínimos para llegar a la reflexión buscada.

Como se visualiza en las transcripciones que realizamos del documento, en la lista de contenidos se refuerza la tendencia hacia los tipos textuales aunque el objetivo declarado sea la enseñanza de los «géneros». Sucede así, nuevamente, lo que Cortés describe sobre la LFE: «pese a la orientación decididamente comunicativa de los CBC de lengua, en general, (...) predominan contenidos y actividades más relacionados con conceptos de la gramática del texto que con los de linguiística textual de orientación comunicativa» (120).

Desde el primer nivel de elaboración curricular hacia el segundo se produjo una reducción notable de contenidos. En la reelaboración jurisdiccional no se seleccionan suficientes contenidos para lograr que las Orientaciones Curriculares, que luego las instituciones utilizarán para realizar la tercera y última reelaboración curricular, adquieran el carácter de «orientaciones». Una problemática que podría presentarse es que, al ser mínimos los contenidos propuestos, las instituciones no tengan posibilidades de selección/reelaboración.

Lo que ocurre en el Nivel Curricular Jurisdiccional de la LEN es similar a lo que ocurrió en la LFE. Aunque el Nivel Nacional contempla de alguna manera en su selección curricular a los contenidos gramaticales, en el siguiente nivel de elaboración curricular no se considera que la enseñanza de la gramática sea uno de los caminos posibles para formar un «sujeto alfabetizado», como afirman Bassano y Freidenberg:

para formar un sujeto alfabetizado, es necesario, (...) «estudiar una lengua» y para estudiarla es imprescindible reflexionar acerca del conocimiento gramatical implícito en todos nosotros como sujetos. (...) es necesario enseñar gramática, concibiéndola no como una mera descripción de estructuras sino atendiendo a la explicación de su buena formación. (94) 
El Eje IV se encuentra vaciado de contenidos, y por lo tanto el lugar del CGV es nulo. La pregunta que surge al leer la inscripción del eje IV es: ¿qué contenidos se encuentran entonces en los ejes anteriores y que abarcan ya al eje IV?

En los ejes I, II y III se detallan temas relacionados con la comprensión y producción oral, la lectura y comprensión escrita y la literatura: texto conversacional, texto argumentativo, texto expositivo, textos mediados por las nuevas tecnologías (chats, correo electrónico, mensajes de texto), textos literarios (teatro, cuento, novela, poesía), textos de opinión, textos periodísticos. Al vaciar de contenidos el eje destinado a la reflexión gramatical se corrobora la premisa que Otañi y Gaspar (2001) anunciaron y que recuperamos en las primeras páginas de este trabajo: la gramática hoy en las escuelas incomoda, o, reformulando la premisa, desde el nivel de elaboración curricular Jurisdiccional no se sabe qué hacer con ella y, en este caso, se la termina expulsando de las OCLyL 2012.

Castellá plantea que la instrucción gramatical puede enseñar a observar, puede sugerir, puede motivar la curiosidad y ofrecer conceptos básicos. Esto no implica la enseñanza de una u otra teoría lingüística sino que instala la necesidad del desarrollo de una "gramática pedagógica» que involucre temas que exijan la participación de más de una teoría lingüística, porque ninguna describe a la lengua en su totalidad.

Desde esta mirada la gramática tiene que ser un soporte, una ayuda, un medio, no un fin. Pero no por no considerarse «el fin» debe excluirse de los contenidos escolares.

El vaciamiento del eje IV se corresponde con la secuencia didáctica propuesta para el trabajo en el aula. En esa secuencia los docentes son los que eligen qué aspectos enseñar de cada género, en el listado de contenidos cada tema propuesto como "género» pauta qué es lo que se espera que se enseñe, aunque estos sean poco claros y no estén desglosados.

Esta ausencia de la gramática en las nuevas propuestas curriculares y la consecuente justificación del lugar marginal que se le otorga puede considerarse lo que Gerbaudo (2011) acuña, enlazando los conceptos de «obstáculo epistemológico» de Bachelard e «ideología» de Eagleton como un «obstáculo ideológico»:

Esta categoría da cuenta de las representaciones que impiden comprender o leer críticamente una situación debido a cristalizaciones de orden ideológico que promueven interpretaciones a priori. Así como la ideología es lo que persuade a hombres y a mujeres a confundirse, de vez en cuando, con dioses o con bichos (Eagleton 15), los obstáculos ideológicos son los que llevan a actuar como «dios» o como «bicho» (es decir sobrevalorando o despreciando una posición que se internaliza al margen de los fundamentos teóricos). (19)

Se evidencia en los documentos un «obstáculo ideológico», no se vuelve a pensar sobre la importancia en la educación de un objeto que ya es despreciado de antemano.

\section{Cuando la reelaboración es un retroceso}

Según lo expuesto en los apartados anteriores el avance que introducen los NAP (2011) en relación con la LFE en cuanto a los contenidos gramaticales es importante y significativo: así como sucedía con los CBC Nacionales de la LFE en los NAP se presenta una lista de contenidos gramaticales aislada del análisis textual y por lo tanto allí se listan contenidos relacionados con la categoría gramatical verbo. 
Pero, nuevamente, tal como ocurrió con los DC de la LFE, este avance es dejado de lado en las reelaboraciones curriculares del segundo nivel: las OCLyL 2012.

En los fundamentos del área lengua y literatura de las OCLyL 2012 se explicita que la gramática es fundamental en la comprensión y producción de textos orales y escritos pero también se afirma que la reflexión gramatical no es el eje de la unidad curricular. Este pasaje reproduce las discusiones instaladas desde la LFE en relación con la enseñanza de la gramática y en consonancia con esa declaración, el eje IV se encuentra vaciado de contenidos. No se listan contenidos relacionados con la gramática y por lo tanto el lugar del contenido gramatical verbo en las reelaboraciones curriculares es nulo.

Recordemos lo que llamó nuestra atención al analizar los NAP. La secuencia didáctica de la LFE en donde la reflexión gramatical que se proponía sólo estaba al servicio de la escritura se reformula en los contenidos de la LEN y en este contexto la reflexión gramatical también apunta a la reflexión en torno a la gramática oracional.

En la reelaboración curricular jurisdiccional parece haber vestigios de los CBC de la LFE y se propone nuevamente una secuencia didáctica en donde la reflexión gramatical es posterior al análisis/producción textual y queda librada al azar. Este hecho es coherente con el objetivo de enseñanza que se reconoce en el documento: «el dominio de los géneros para la participación en la vida socio-comunitaria otorgando status de apoyo técnico a los aprendizajes de la sintaxis y el léxico» (OCLyL 2012:37).

Este giro de las OCLyL 2012 con relación a los NAP vuelve a poner en el lugar de normativa a la gramática. Otañi y Gaspar (2001) argumentan que en la LFE se concebía a la gramática como un conjunto de reglas a seguir para producir textos correctos y adecuados. En esta línea, en los documentos curriculares de la provincia de Santa Fe ya no se la concibe, ni siquiera, como un conjunto de reglas a seguir para la buena formación de los textos. La reflexión gramatical se ubica, nuevamente y si el docente así lo decide, en el lugar de un conocimiento ocasional.

Para cerrar este segundo apartado recuperamos de Ciminari y Kocak una analogía que pone en evidencia la necesidad de que los alumnos se interioricen en el conocimiento gramatical:

Imaginemos un arquitecto que pretenda construir un edificio sin saber nada de ladrillos y cemento. Por más que justifique el aspecto estético de su obra, no podrá evitar que se derrumbe por mala elección de material. Lo mismo ocurre cuando un niño o adolescente quiere construir un texto para comunicar su mundo interior. (171)

\section{Reordenando el andar: el quehacer docente}

Las propuestas didácticas del campo educativo concreto que tomamos como muestra pertenecen al $1^{\circ}$ año de la Educación Secundaria Obligatoria (ESO) de la ciudad de Santa Fe del ciclo lectivo 2013. Este recorte se justifica en que las primeras OCLyL que analizamos, la versión 2012, contempla el desarrollo curricular sólo de primer y segundo año de la ESO. Recortar estas propuestas didácticas al ciclo lectivo 2013 nos permitió indagar en las respuestas concretas de los docentes a la situación existente: la aparente ausencia de la reflexión sobre la lengua en las propuestas educativas oficiales.

De las propuestas de aulas de lengua seleccionadas como muestra centramos nuestra atención en los contenidos pautados en los programas escritos, contenidos que son pensados para ser 
desarrollados durante el ciclo escolar. De la enumeración de los contenidos nuestro interés está direccionalizado hacia el CGV.

Siguiendo a Lorenzotti y Carrió entendemos por contenidos a:

aquello que se enseña. Los «contenidos» son los conceptos, categorías, operaciones, deducciones, explicaciones, que efectivamente se enseñan, que no se dan por supuestos, que no se espera que el alumno deduzca solo. Los «contenidos» son aquellos conocimientos para los que se requieren instrucción, teorización, en el sentido de que el alumno no puede saberlo sin que el otro se lo enseñe. Los «contenidos» son los "conceptos específicos» que se desarrollan en el aula y que se espera que el otro aprenda genuinamente para activarlos en el momento en que lo requiera. Esto es, lo que se «enseña» en el aula independientemente de lo que se «aprenda». (47)

Las propuestas escritas nos proporcionan datos sobre un listado de contenidos posibles diseñados, en la mayoría de los casos, sin un diagnóstico previo de los alumnos del ciclo. Analizamos estas propuestas con la intención de rastrear qué sucedió con el CGV anulado del documento oficial de la jurisdicción, junto con todo el eje vinculado con los contenidos gramaticales. De este análisis se desprende que las propuestas se construyen con muy pocas continuidades y muchas rupturas-reconstrucciones de la ausencia de los documentos jurisdiccionales.

Entendemos por continuidad de la ausencia a la permanencia de la propuesta OCLyL 2012 en las propuestas de los docentes. De las quince propuestas trabajadas como muestra sólo una continúa linealmente con la selección curricular del documento jurisdiccional. En esa propuesta se continúa tanto con la estructura organizativa, esto es la división en los cuatro ejes, como con los contenidos listados, y a partir de esta continuidad el CGV está ausente de la propuesta.

En otras tres propuestas más se reproduce la continuidad de la ausencia del CGV, pero a partir de las entrevistas con los autores de estas propuestas pudimos constatar que si bien el CGV no aparece formalmente enunciado en los documentos escritos sí se trabaja con él en la propuesta áulica, en el desarrollo de otros contenidos del área, forma parte del «curriculum oculto» (Morzán) de la enseñanza.

En esas propuestas el contenido es abordado en el aula cuando se trabaja con «clases de palabras» con la finalidad de distinguirla de otras como el adjetivo y el sustantivo; también se trabaja sobre su función en la sintaxis oracional (núcleo del predicado); el uso de los tiempos verbales en los textos con secuencia narrativa (crónicas, cuentos) y finalmente su función en los textos instructivos (el infinitivo y el modo imperativo). Se aborda el contenido entonces tanto como clase de palabra como desde su función en la oración y en los textos.

Es decir que si bien en las propuestas escritas, en los documentos institucionales, no se enuncia explícitamente como contenido al verbo sí se lo trabaja en momentos específicos del desarrollo curricular en relación con otros contenidos del programa.

Este acercamiento por fuera del curriculum que reconocen los docentes autores de las propuestas que se enmarcan en las continuidades es similar al tratamiento del CGV que le dan las demás propuestas, las que rompen-reconstruyen los contenidos oficiales.

Por ruptura consideramos al quiebre de relaciones con las OCLyL 2012 de las propuestas de los docentes. Consideramos que rompen con la propuesta oficial aquellas planificaciones que no siguen los lineamientos curriculares de las orientaciones jurisdiccionales, que se alejan tajantemente de las nuevas disposiciones, pero que a la vez, a partir de ese alejamiento, reconstruyen la ausencia. 
Las propuestas que rompen-reconstruyen son las que diagraman la selección de contenidos a partir de otros múltiples aportes. Las propuestas recuperan de las OCLyL 2012 los aspectos que consideran pertinentes para la formulación de los contenidos, los diferentes ejes de trabajo, pero se diagraman en función de otros contenidos pertenecientes, en la mayoría de los casos a la LFE. Estos contenidos pueden considerarse los resabios, lo que queda del cambio curricular anterior, pero en la mayoría de los casos pertenecen a los documentos nacionales y no jurisdiccionales.

Los docentes autores de las propuestas pensaron y diagramaron sus propuestas de aulas de lengua para el primer año de la ESO del Ciclo Lectivo 2013 incluyendo en ellas al CGV. Esta inclusión marca un alejamiento, un corrimiento de las OCLyL 2012 y los contenidos curriculares con los que se reconstruyen las propuestas pertenecen, en la mayoría de los casos, a la Ley de Educación anterior. Esto demuestra que la nueva propuesta enmarcada en la LEN no tuvo grandes repercusiones al interior de las propuestas docentes. Detectamos pocas relaciones y la mayoría de ellas ancladas a la incorporación como contenido de las nuevas tecnologías: chats, mensajes de texto, aulas virtuales, correo electrónico.

Las propuestas que incluyen al CGV marcan un distanciamiento de la propuesta jurisdiccional de la LEN. Al no estar explicitado qué contenido gramatical enseñar en las Orientaciones Jurisdiccionales, cuando en las propuestas de aulas de lengua se incluye al CGV se lo hace, en la mayoría de los casos, retomando los contenidos del Nivel Nacional de la LFE. Al hacer esta incorporación se vincula al contenido con la función que desempeña en los textos o con su función sintáctica como clase de palabra.

Si bien se procura un espacio en muchas de las propuestas para el tratamiento léxico del CGV y para la reflexión gramatical, muchas veces esta reflexión se ve boicoteada por otras urgencias como ser la corrección textual.

De este modo, la aparente articulación entre los documentos del docente y los documentos oficiales de las nuevas propuestas curriculares termina convirtiéndose en una des-articulación. Esto es porque las piezas ordenadas que conforman una propuesta curricular oficial, ya sea de la LFE o de la LEN, son desmanteladas por el docente autor y constructor de su propuesta. El docente separa, altera, desorganiza las piezas, selecciona los contenidos sugeridos que considera indispensables para su «buena propuesta» y las articula con otros contenidos ya des-articulados de propuestas educativas anteriores, en vistas a saturar los objetivos para su aula.

A partir de esta des-articulación el CGV no queda ausente de las propuestas docentes, es recuperado de propuestas oficiales pertenecientes a períodos anteriores, en este caso a la LFE, y reacomodado en función de los objetivos del presente. La problemática que esto acarrea es que tanto los contenidos como su abordaje que se recuperan de otras propuestas se cristalizan en las planificaciones docentes. Estas cristalizaciones responden a otros enfoques para la enseñanza de la lengua, así las des-articulaciones llaman a la convivencia dentro de las propuestas docentes de variadas propuestas oficiales recortadas del contexto en el que significan.

\section{Discusión}

A lo largo de este trabajo realizamos un recorrido en busca de respuestas a tres interrogantes de base. Desde el principio analizamos el lugar del CGV en las reelaboraciones jurisdiccionales de la LEN movilizados por una ausencia que consideramos preocupante para el «aula de lengua». 
Luego de realizar el recorrido por los cambios curriculares de nuestro país detectamos que la tendencia de la jurisdicción Santa Fe es la de omitir los contenidos gramaticales que sí se listan en el nivel nacional. Quizás esta tendencia haya sido ya un foco de preocupación para los docentes santafesinos lo que acarreó que en sus propuestas las ausencias no se hagan visibles y que la construcción de las mismas esté des-articulada con las propuestas jurisdiccionales oficiales.

Así como lo define De Alba entendemos que el «curriculum» se construye a partir de luchas entre intereses «diversos y contradictorios».

Entendemos que estas luchas se producen a medida que se realizan las reelaboraciones de los diferentes niveles.

Entendemos que los docentes «intelectuales transformativos» son el eslabón final de esas luchas.

Celebramos la iniciativa del ministerio provincial de generar instancias de revisión curricular a partir de la implementación de las orientaciones borradores aunque luego de nuestro acercamiento a los docentes concebimos que esas instancias no fueron ni suficientes ni significativas para ellos.

Desde esta perspectiva consideramos que es necesario potenciar el lugar que ocupan los docentes en los procesos de determinación curricular. Se necesita brindarles los espacios para que actúen como intelectuales y transformativos antes de que los cambios sean efectivamente implementados, para que no se piense en su accionar, en términos de Saleme, como un "peón» sino como un docente «transformador»:

Si un peón de ajedrez no sabe que puede neutralizar al rey, no lo jaquea. Un docente con oficio de peón de la cultura no domina estrategias de conocimiento, si bien en el terreno concreto de su acción resuelve pragmáticamente situaciones. No enseña más que lo que le enseñaron y tal como lo aprendió. No toca la raíz problemática de su campo. Si estuviera preparado para hacerse preguntas en lugar de perseguir respuestas, podría interpelar al objeto de conocimiento y al conocimiento mismo en tanto éste es objeto de conocimiento, sin abandonar por ello la tarea específica, ni la condición docente. (68)

La realidad escolar no es sencilla, los docentes no siempre tienen la posibilidad de convertirse en los autores de sus propuestas. Las normas institucionales muchas veces pautan que los nuevos docentes simplemente hereden las propuestas de sus aulas de lengua de otros docentes que realizaron su propia selección curricular o, muchas veces, las propuestas son construidas por otros miembros de la institución educativa.

En muchos casos, las propuestas se piensan cristalizadas y la problemática que se plantea es que las planificaciones no se re-elaboran basadas en los cambios curriculares sino que se completan con lo nuevo que se aparece en los cambios.

Consideramos que el CGV, si bien está presente en las propuestas de aulas de lengua, está opacado. Esto se debe a que su enseñanza está en la mayoría de los casos vinculada con los contenidos textuales. En muchos de los casos la reflexión gramatical sobre el verbo es posterior al análisis textual y este análisis está pensado desde una propuesta educativa caduca lo que lo relega al lugar de normativa, al lugar de reglas a cumplir o a respetar en la construcción de determinados textos.

Sería importante reconsiderar el lugar que se le asigna a la CGV en las propuestas oficiales. Diversos trabajos, entre ellos Defagó, discuten que en los años posteriores a la implementación 
del sistema comunicativo la atención puesta en el texto no mejoró el nivel de producción y comprensión textual de los alumnos, uno de los grandes objetivos del área.

Por otro lado, en los demás casos estudiados, se incluye a la CGV restringida al lugar de núcleo del predicado en el análisis oracional, muchas veces sin una reflexión gramatical previa del verbo. La incorporación del contenido se produce en el aula con vistas a actividades de identificación, de rotulación de estructuras. Al respecto, coincidimos con Defagó y Guglielmelli en que con este tipo de actividades «no se aprende o capta cómo funciona la lengua, sino un nuevo vocabulario o etiquetas que obstaculizan la reflexión metalingüística indispensable para después guiar la reflexión metacognitiva vinculada con la producción y comprensión textual».

Para llegar a reflexionar sobre la construcción de los textos es necesario objetivar el lenguaje para crear un metalenguaje compartido entre alumnos y docentes. Es necesario poseer un conocimiento compartido para que la enseñanza no sea contenidista. Para que el conocimiento no se vuelva un «conocimiento ritual» (Perkins). El «conocimiento ritual» es evidencia de que el conocimiento es «frágil». En la mayoría de los casos el análisis de oraciones tiene con frecuencia en las aulas un carácter repetitivo. Este carácter repetitivo sin explicación es lo que se convierte para los alumnos en conocimiento ritual, un conocimiento que sólo sirve «para cumplir con las tareas escolares» (37).

Desde esta propuesta sostenemos la concepción de gramática explícita como herramienta que permite potenciar la reflexión sobre el lenguaje.

\section{Notas}

1 Parafaseando a Roger Chartier.

2 Considerando «lugar» tanto al momento de inserción del contenido en las propuestas, como a su inclusión misma -es decir, secuencia didáctica y secuencia epistemológica.

$3 \mathrm{Al}$ hablar de "propuestas de aulas de lengua» hacemos referencia a lo que en diferentes momentos históricos y desde distintos marcos de discusión se denominó/a: «planificaciones»; "programas»; «itinerarios»; «recorridos didácticos»; «secuencias didácticas».
4 Cabe destacar que en las reelaboraciones jurisdiccionales el espacio curricular se denomina «Lengua y Literatura», se le da el lugar en la nomenclatura al objeto literatura, ausente en los NAP (2006; 2011).

5 Entre mayo y junio del año 2012 se conformó una comisión ad hoc a la que fueron invitados profesores de institutos de formación docente y de universidades nacionales para discutir y revisar los contenidos de las Orientaciones Curriculares para el Ciclo Básico de la Educación Secundaria (2012).

\section{Bibliografía}

Alba, Alicia de (1991). Curriculum: crisis, mito y perspectivas. México: Universidad Nacional Autónoma de México.

Alvarado, Maite (Coord.) (2001). Entre líneas. Teorías y enfoques en la enseñanza de la escritura, la gramática y la literatura. Buenos Aires: Manantial, 2009.

Bajtín, Mijail (1979). Estética de la creación verbal. México: Siglo XXI, 1982.

Bassano, Marcela y María Susana Freidenberg (2004). «Enseñar gramática en la lengua materna». Centro de

Estudios del Lenguaje UNR. Estudios del Lenguaje y enseñanza de la Lengua. Venado Tuerto: Juglaría, 89-139.

Bombini, Gustavo (2001). «Avatares en la configuración de un campo: la didáctica de la lengua y la

literatura». Lulú Coquette. Revista de didáctica de la lengua y la literatura (1)1, 24-33.

Camilloni, Alicia (Comp.) (2007). El saber didáctico. Buenos Aires: Paidós, 2011. 
Carrió, Cintia (2005). «El debate en torno a la enseñanza de la lengua: el lugar de la gramática». Actas del IV Congreso de las Lenguas del MERCOSUR. Lenguaje y Multidisciplinariedad. Chaco: Universidad Nacional del Nordeste, $150-157$.

(2013). «Ni trabalenguas ni trabas en lengua: son de los que no son... Ana, aunque crezca, no será anón». El toldo de Astier. Web.

Castellà, Josep (1994). «¿Qué gramática para la escuela? Sobre árboles, gramáticas y otras formas de andarse por las ramas». TEXTOS de didáctica de la lengua y la literatura 2, 15-24.

Ciapuscio, Guiomar (1994). Tipos textuales. Buenos Aires: Eudeba.

Ciminari, Luciana y Claudia Kocak (2004). «El contenido ausente: léxico/estructuras. Segunda Parte: Léxico y estructura: develar el contenido ausente». Centro de Estudios del Lenguaje UNR. Estudios del Lenguajey enseñanza de la Lengua. Venado Tuerto: Juglaría, 168-189.

Cortés, Marina (2001). «Los textos. Marcos teóricos y prácticas de enseñanza», en Maite Alvarado, coordinadora. Entre líneas. Teorías y enfoques en la enseñanza de la escritura, la gramática y la literatura. Buenos Aires: FLACSO/Manantial, 2009, 113-144.

Defagó, Cecilia (2003). «Lo simple no es siempre lo más sencillo. Una reflexión sobre la gramática en la Escuela». Actas del IV Congreso Nacional de Didáctica de la Lengua y la Literatura. Córdoba: Universidad Nacional de Córdoba.

Defagó, Cecilia y Jorge Guglielmelli (2005). «La reflexión gramatical. De lo implícito a lo explícito en la enseñanza de la lengua». II Jornadas Internacionales de Educación Lingüística. Concordia: Universidad Nacional de Entre Ríos.

Demonte, Violeta (2005). «La gramática ubicua. O cómo se miran los textos a través de la gramática». Universidad Autónoma de Madrid, Instituto Universitario Ortega y Gasset. Web.

Fenstermacher, Gary (1986). «Tres aspectos de la filosofía de la investigación sobre la enseñanza», en Merlin C. Wittrock, editora. La investigación de la enseñanza, I. Enfoques, teorías y métodos. Buenos Aires: Paidós, 1995, 149-179.

Flinders, David y otros (1986). «Curriculum nulo: su base teórica y sus implicancias prácticas». Curriculum Inquiry, 16(1), 33-42.

Gerbaudo, Analía (2006). Ni dioses ni bichos. Profesores de literatura, currículum y mercado. Santa Fe: Universidad Nacional del Litoral.

(2011). «El docente como autor del currículum: una reinstalación política y teórica necesaria». La lengua y la literatura en la escuela secundaria. Santa Fe/Rosario: Universidad Nacional del Litoral/Homo Sapiens, 17-27.

Giamatteo, Mabel y Hilda Albano (2009). ¿Cómo se clasifican las palabras? Buenos Aires: Biblos.

Giroux, Henry (1990). Los profesores como intelectuales. Barcelona: Paidós, 1997.

Litwin, Edith (1996). «El campo de la didáctica: la búsqueda de una nueva agenda», en Alicia Camilloni y otros. Corrientes didácticas contemporáneas. Buenos Aires: Paidós, 2010, 91-116.

Llambí de Adra, María Eugenia (2005). «Aportes para redefinir el valor del léxico en la enseñanza de la lengua». Web.

Lorenzotti Micaela y Cintia Carrió (2015). «Contenidos y objetivos: dis-continuidad en el área de Lengua y Literatura de los documentos Jurisdiccionales (Santa Fe, 2012-2013)», en Cecilia Muse, editora. Currículo, evaluación y formación en lectura y escritura. Córdoba: Universidad Nacional de Córdoba, 45-54.

Morzán, Alejandra (2007). «Currículum y enseñanza». Saberes y sabores de la práctica docente. Textos y contextos. Resistencia: De la Paz. 
Otañi, Isabel y María del Pilar Gaspar (2001). «Sobre la Gramática», en Maite Alvarado, coordinadora. Entre líneas. Teorías y enfoques en la enseñanza de la escritura, la gramática y la literatura. Buenos Aires: FLACSO/ Manantial, 2009, 75-111.

(2008). «Gramática, lectura y escritura: Aportes para redefinir el lugar de la gramática en la escuela». Web.

Perkins, David (1992). La escuela inteligente. Del adiestramiento de la memoria a la educación de la mente. Barcelona: Gedisa, 1998.

Puiggrós, Adriana (2003). Qué pasó en la educación argentina. Breve historia desde la Conquista hasta el Presente. Argentina: Galerna.

Riestra, Dora (2010). «Para debatir: ¿Cómo surgen y cómo llegan las propuestas didácticas?». El toldo de Astier, propuestas y estudios sobre la enseñanza de la lengua y la literatura (1)1, 1-15.

Saleme de Burnichon, María (1997). «Una relación casi olvidada: el docente y el conocimiento». Decires. Córdoba: Narvaja. 67-71.

\section{Documentos curriculares}

Diseño Curricular Jurisdiccional para el Tercer Ciclo de la EGB, para Lengua y Lengua extranjera, Ministerio de Educación de la Provincia de Santa Fe, 1999.

Núcleos de Aprendizaje Prioritarios. Ciclo Básico Educación Secundaria $1^{\circ}$ y $2^{\circ} / 2^{\circ}$ y $3^{\circ}$ años. Lengua. Consejo Federal de Cultura y Educación. Ministerio de Educación, Ciencia y Tecnología. Buenos Aires. Octubre 2011.

Orientaciones Curriculares para el Ciclo Básico de la Educación Secundaria, Ministerio de Educación de la Provincia de Santa Fe, versión febrero de 2012.

Orientaciones Didácticas. Tercer Ciclo EGB. Lengua. Documento de desarrollo curricular. Ministerio de Educación de la Provincia de Santa Fe 1997. 